\title{
Nudging the Classroom: Designing a Socio-Technical Artifact to Reduce Academic Procrastination
}

\author{
Joaquin Rodriguez \\ Louisiana State University, \\ jrod122@1su.edu
}

\author{
Gabriele Piccoli \\ Louisiana State University, \\ gpiccoli@1su.edu
}

\author{
Marcin Bartosiak \\ University of Pavia, \\ marcin.bartosiak@unipv.it
}

\begin{abstract}
Procrastination is a widespread detrimental human behavior. Virtually everyone delays the initiation or completion of important tasks at times. Some people procrastinate to the point that they become overwhelmed by their inaction. In particular, academic procrastination is estimated to afflict 70 to $90 \%$ of undergraduate college students. We adopt the design science problem-solving paradigm to pilot a socio-technical artifact that reduces academic procrastination in large college classrooms. We adopt the principles of nudging to propose three meta-requirements and nine design principles underlying the design of a chatbot that induces students into positive and self-reinforcing behaviors countering procrastination tendencies. We use a formative natural evaluation event to provide preliminary validation for the design. The pilot provides encouraging results both in terms of use of the artifact by the intended audience and of performance improvement and can therefore be used to inform future design iterations.
\end{abstract}

\section{Introduction}

Humans engage in a wide range of undesirable behaviors (e.g., smoking, unhealthy eating, compulsive gambling). The paradox is that individuals pursue such activities despite being conscious of their negative consequences. Humans are predictably biased. We behave in predictable non-rational ways when confronting specific types of decisions [1]. For instance, individuals tend to favor short-term gratifications [2], often postponing the start of a healthy diet or the completion of tedious paperwork. These are acts of procrastination that hamper successful performance, in our personal, academic, or professional life.

We define procrastination as the act of delaying initiation or completion of important tasks to the point of discomfort [3]. Virtually everyone procrastinates, to a greater or lesser extent, in their everyday life [4].
For example, the United States is the country with the highest number of overweight adults in the world at nearly $70 \%$ of the population. Yet, virtually everyone knows that healthier eating habits and regular exercise can improve their condition. Most people struggle to break detrimental habits [5].

Academic procrastination among college undergraduates reaches alarming rates. By some accounts, $70-90 \%$ of undergraduates procrastinate at school $[6,7]$. Procrastination is especially damaging in college, a high-demanding environment where students are held to firm deadlines and evaluations. Furthermore, like many other life's decisions, college is a "low practice" endeavor. Most students get only one chance to make their college experience successful.

Typically, studies on academic procrastination focus on understanding its causes and consequences [8]. However, it is difficult to precisely identify antecedents and outcomes as internal norms regulate the causal relationships of procrastination. The construct is associated with several negative effects, such as low academic performance, low self-efficacy, high levels of anxiety, and depression [8]. Furthermore, procrastination is associated with low intrinsic motivation [6], which is particularly worrying in the college environment as presumably, students enroll in courses that are preparatory to their future careers.

As research focuses on addressing the antecedents and outcomes of procrastination, little work has focused on designing and implementing effective strategies to decrease this detrimental behavior. We take a proactive stance grounded in the design science research paradigm. Our research objective is to implement a Socio-Technical (ST) artifact [9] that promotes positive self-reinforcing behaviors in order to persuade students in an introductory Information Systems course to reduce their procrastination tendencies. We are not aware of any existing ST artifact designed to proactively reduce academic procrastination at scale. We, therefore, claim an "improvement" knowledge contribution, focused on developing new solutions for known problems [9, 
p. 345]. Preliminary evaluation of the artifact in a pilot setting shows that procrastination among students is reduced.

\section{Problem Definition}

When students postpone the initiation or completion of important academic activities until the proximity of the deadline, they end up with limited time to complete the task. The spacing effect demonstrates how when humans learn over spaced sessions rather than concentrated ones, their mastery of the material improves [10]. However, when students procrastinate, they limit their chances to iterate multiple times over the subject matter, which ultimately leads to low retention, lower learning performance, and lower evaluation marks $[8,10]$. Furthermore, procrastination is associated with a number of detrimental psychological effects, such as low self-esteem, anxiety, and depression $[8,11]$.

Academic research measures procrastination through self-reports, such as the Procrastination Scale [12] or the Procrastination Assessment Scale [13]. However, the identification of procrastination remains problematic, given its nature as an intra-individual process. For example, what for some is procrastination, for others it is a purposely planned delay. Some researchers differentiate between passive and active procrastinators. Where the former are procrastinators in a traditional sense, the latter can be thought of as "strategic procrastinators" - individuals who deliberately delay their actions to focus on other important tasks. Active procrastinators tend to be in full control of their actions and like to work under pressure [14]. Thus, we focus on procrastination as the intra-individual process of postponing the initiation of an intended course of action to the point where the individual is overwhelmed by their inaction [8].

Human relationships between peers and between the instructor and the students are critical for high-quality education outcomes [15, 16, 17]. Instead of using grades, effective instructors motivate students by reinforcing the "payoff" of knowledge acquisition while providing personalized and recurrent feedback $[18,19]$. This approach reduces strategic learning [18] while increasing students' intrinsic motivation [20].

Increasing resource constraints in universities put pressure on course delivery by steadily increasing classroom sizes [21]. Sometimes, human contact is completely removed as courses are virtualized into massive open on-line courses (MOOC) [22] or virtual learning environments (VLE) [23].

Universities should enable young people to achieve their maximum potential through subject matter learning and the development of strong learning habits. Yet, increasingly large classes challenge this mission and result in lower subject matter mastery [24], particularly for students who are at the top of the grade distribution [25]. Interaction and engagement decrease in large classrooms where students remain anonymous, leading to a lower motivation for both faculty and students [26] and increasing detrimental behaviors, such as procrastination [27]. Under resource constraints, professors struggle to create personal connections with students, understand their strengths and weaknesses, provide personalized feedback or promote positive behaviors. Resource constraints contribute to detrimental habits, such as procrastination, that produce negative consequences on students' well-being and performance [27]. As the size of the classrooms is unlikely to decrease in the foreseeable future, this class of problems will persist. Thus, there is a need for innovative solutions that can support and promote positive students' behavior "at scale."

Our work focuses on an introductory course in a business school in the USA. The course, titled "Introduction to Management and Information Systems", is required for all first-year business and economics majors. The course covers both theoretical and practical topics. The former includes the foundations of information technology and information systems in business. The latter focuses on proficiency with Microsoft Office productivity tools (e.g., Microsoft Excel). In a typical year, over 1,500 students enroll in the course, divided into sections of about 200.

While we contextualize the design of the artifact in the context of an introductory information systems course, we aim to inform solutions for a class of problems [28]: the design of a ST artifact [29, 9] that reduces academic procrastination in large college courses, under resource constraints.

\section{Nudging the Classroom}

We propose that instructors, in order to overcome the impossibility to meaningfully interact with a large number of students, should leverage information technology to nudge learners into positive and self-reinforcing behavior. We follow the design science approach to uncover design principles (DP) for an ST artifact [29, 9] that reduces academic procrastination. The problem-solving approach of design science supports our goal of contributing to the scholarly knowledge base by designing, building, and testing a working solution [30].

We follow the iterative design science research methodology (DSRM) [31]: 1) identify problem and 
motivate; 2) define objectives of the solution; 3 ) design and development; 4) demonstration, 5) evaluation, 6) communication. In this paper, we ground the research in an important class of problems, define clear objectives for the solution, and discuss the design and development of the artifact. The design of the ST artifact is guided by meta-requirements (MR) [32] derived from a theoretical framework (i.e., kernel theory) and the analysis of the currently available technologies on the market. We also demonstrate a first implementation of the ST artifact design. We use a formative natural evaluation event [33] to provide preliminary validation for the design. This report of an ongoing project is, therefore, an "empirical contributions that can become [a] building block in a journey towards theoretical contribution." [34, p. 599]

\section{Objectives of the Solution}

Research has investigated the causes for academic procrastination in relationship with personality traits, self-regulation, self-efficacy, and self-esteem [6, 35, 36]. In particular, the association between self-efficacy and procrastination has received great attention as self-efficacy - a person's beliefs in his capabilities to execute behaviors that would achieve a desired outcome [37] - emerged as an important predictor of procrastination [38, 39, 40]. However, "knowing what to do is only part of the story" [37, p. 223]. Motivation is another important factor affecting both self-efficacy and procrastination [41, 42]. For example, students that are intrinsically motivated tend to procrastinate less than students that are extrinsically motivated [42].

Most research on procrastination focuses on its antecedents and consequences and solutions to overcome it are still rare. One of the few exemptions is Piers Steel's book [43], where the leading scholar on the subject provides effective strategies to decrease self-destructive habits. However, the strategies provided are not aided by technology and require strongly motivated individuals who recognizing the detrimental effects of procrastination voluntarily intervene to improve their situation.

\section{Theoretical Framework}

Nudging is the kernel theory from which we draw meta-requirements and design principles for our ST artifact's design. While our work might generalize beyond education, there are a number of context specific characteristics to consider. First, the learning process is more effective when students are intrinsically motivated to internalize cognitive and skill-based outcomes [44]. Although we can influence motivation through extrinsic rewards, such as a grade, research found that the effects of extrinsic motivation tend to decrease over time and might negatively affect intrinsic motivation $[45,46]$. Second, the classroom setting should promote the success of all its participants regardless of their traits, motivation, or previous knowledge. Recent research found that learners in gamified contexts report high levels of self-efficacy and learning outcomes when faced with a lower-skilled competitor [47]. However, although this outcome is beneficial for the higher-skilled individual, the lower-skilled one is subjected to negative self-efficacy impacts. There should not be winners and losers in the classroom. Fourth, there exist privacy concerns about the achievement results among students. In the educational context, privacy concerns are not only related to students' feelings but also to laws regulating educational records disclosure [48]. Fifth, the length of the learning process requires recurrent interactions with the subject material. Furthermore, these interactions are spread over a significant time period (e.g., 4-5 months in the college context). Conversely, extrinsic rewards are optimal when the user interacts infrequently with the system over a short period of time [49]. Lastly, students adopt multiple strategies in their learning process [50]. Introducing incentives to modify their behavior (e.g., completion badges) can induce actions that might not be aligned with students preferred learning style. For example, rewards (e.g., extra points) can be assigned for the completion of activities rather than for mastering the material. In extreme cases, when the learning is embedded in stimulating game environments through gamification, students might pay more attention to the game itself than to the learning [51].

We argue that the design of a ST artifact to reduce academic procrastination should maintain or increase freedom of choice. However, humans do not always make the right choices when they are not experienced with the available options, do not have enough information, or are required to process numerous options [52]. In the context of college, all three of these conditions typically hold.

College students need to adapt to a context where the learning process requires more self-discipline, and where their initial network of close relationships is quite limited or non-existent. Furthermore, for many students, going to college constitute their first experience living away from home.

Students often evaluate their choices with limited information. For instance, when students are not aware of the "payoff" of the learning activities they can easily lose interest in the course [27]. More simply, often students are unaware of the required deadlines or commitments. 
The many activities that students undertake, or can potentially undertake, during the academic semester can become overwhelming. As humans have limited attention [1], we are not necessarily aware of all the activities we can or should engage in at a specific moment to maximize our long-term well-being. As the number of possible activities increase (e.g., go out with friends, go to the gym, do homework), the probability that students will undertake beneficial behavior decreases.

We therefore adopt a libertarian paternalism approach that embraces the concept of digital nudging. Libertarian paternalism, a term coined by Nobel Prize winner Richard Thaler, is the notion that a designer "attempts to influence the choices of affected parties in a way that will make choosers better off" [53, p. 4]. Under libertarian paternalism individuals still enjoy freedom of choice, but are nudged toward choices that are more beneficial. In this context, a nudge is "any aspect of the choice architecture that alters peoples behavior in a predictable way without forbidding any options or significantly changing their economic incentives." [52, p. 6] The choice architecture is the context in which people make decisions. Although nudges have been studied primarily in off-line contexts, the use of digital nudges can affect both digital and real-world behavior [54]. Five principles guide the design of nudges: incentives, understanding mappings, defaults, giving feedback, expecting error, structure complex choices [55].

Incentives - Nudges focus on improving the salience of the incentives already present. In fact, even when strong economics incentives are in place (e.g., peak hour energy surcharges) humans might ignore them. Nudges, by making the incentives more salient, increase their effectiveness. Understanding mapping - Where humans lack experience, they tend to have difficulties in understanding the relationship between their choices and their resulting welfare. Improving the understanding of the mapping between individual choices and results can improve the chances of making good decisions. Defaults - Choice architectures are never "neutral" as the context is always influenced by choices and non choices (e.g., randomly display of products) of the designers. Nudging proponents argue that designers should promote by default positive and beneficial behavior. Feedback - Providing feedback to individuals when they perform or under-perform has been shown to improve performance. For instance, frequent positive verbal feedback tends to foster students' intrinsic motivation [19]. Expecting error - Humans are naturally inclined to make mistakes. A well-designed system should account for and expect users to err. Structure complex choices - When choices depend on numerous and complex available options, humans adopt simplifying strategies and heuristics to cope with the ensuing overload. A good system should structure the attributes of all the alternatives in a meaningful manner to facilitate trade-off analysis and simplify the decision process.

\section{Designing the ST Artifact}

We conceptualize our solution as the design of a socio-technical (ST) artifact [29] where social and technical design features play equally important roles. Following the principles of nudging we advance meta-requirements and design principles for the ST artifact design.

Traditionally, in a classroom environment students are not subjected to classic economic incentives ${ }^{1}$. Instructors have only one main extrinsic motivator at their disposal: grades. Moderate levels of extrinsic motivation, through grades, can produce positive effects when coupled with high levels of intrinsic motivation [44]. However, grades are ineffective and encourage procrastination when they do not reflect student achievement, such as when grade inflation is observed [27, 44]. Furthermore, students tend to easily lose interest in the course when the required learning activities are not perceived as relevant to their future careers [27], such as when incentives are provided for activities that do not necessarily improve learning. Therefore, as prescribed by nudging principles [52], the ST artifact should not introduce incentives that distort students' behavior. Instead, the artifact should promote positive behavior independently, just as successful instructors do [18].

MR1: The ST artifact for procrastination reduction must not conflate behavior with learning.

- DP1.1: Student activities and behaviors (e.g., attendance) have no bearing on the students learning assessment (i.e., the grade).

- DP1.2: Assignments and homework are a service to students and have no bearing on the students learning assessment.

- DP1.3: Learning assessment is measured, independently of student behavior, through dedicated ad-hoc evaluations (i.e., exams).

Students often feel guilty and believe that they should have worked harder in their classes [27]. However, even though students are at least partially

\footnotetext{
${ }^{1}$ Performance-based scholarship presents an exception.
} 
aware of the fact that procrastination is detrimental, they still engage in it. Thus, a ST artifact that reduces academic procrastination must intervene proactively to nudge students into appropriate actions. Specifically, the ST artifact should provide a choice architecture that encourages students into positive and self-reinforcing activities by default.

The mediation of computers in everyday activities [56] enables the use of IT as a medium of persuasion [54]. Moreover, the use of technology is essential to design interventions under resource constraints, because designers can capitalize on the scalability afforded by software. One of the most noteworthy cases of digital nudge at scale is the health features of the Apple Watch, which gently reminds users to perform behaviors leading to a healthier lifestyle.

Digital nudges can assume many forms. For instance, displaying healthy options first in online food ordering menu is a subtle nudge. Notifications reminding you to go to the gym are more direct prompts. Many successful digital nudges, as in the Apple Watch example, adopt triggers. Triggers are calls to actions that prompt a specific behavior [57]. The notifications that we receive on our phone, such as real-time traffic alerts, are triggers. Previous research demonstrates that triggers in the form of email reminders can reduce procrastination of learners [58]. The literature distinguishes three different types of triggers: sparks, facilitators, and signals [57]. Spark triggers are best to motivate behavior (e.g., persuade citizens to evacuate a city threatened by an approaching hurricane). Facilitator triggers enable a behavior by reducing barriers to action (e.g., a one-click flight check-in). Signal triggers are more appropriate as reminders or to indicate a behavior (e.g., reminder to take medications).

MR2: The ST artifact for procrastination reduction proactively triggers appropriate behaviors.

- DP2.1: Adopt signal triggers to remind students of deadlines and commitments (e.g., assignment deadlines).

- DP2.2: Adopt spark triggers to alert at-risk students and urge them to action.

- DP2.3: Adopt facilitator triggers to reduce obstacles to performing appropriate behaviors (e.g., prompting a question of the day through a conversational interface).

In line with nudging principles, positive behavior encouraged through default triggers should only impose a minimal cost on students. The simplicity and timing of triggers are critical factors mediating their success [57].
Trigger effectiveness increases with their immediacy and timing fit to the target behavior [57]. Conversely, prompting an action when recipients are unable to act causes detrimental frustration [57, 59]. Personalization can significantly influence the efficacy of systems as it improves personal relevance, motivation, engagement and self-efficacy [60, 61, 62]. Therefore, the ST artifact should encourage sustained use by providing some degree of personalization.

MR3: The ST artifact for procrastination reduction encourages sustained use by managing triggering risks.

- DP3.1: Signal triggers are contextually aware (e.g., reminders are targeted).

- DP3.2: Students can customize the acceptable triggering window (e.g., time of day) or suspend triggers.

- DP3.3: Students can manage the type of triggers they receive (e.g., Requesting a "question of the day").

\section{Artifact Implementation}

Our team implemented and pilot tested a first iteration of the ST artifact during the 2018 spring semester. As a first implementation, the objective was to test the viability of the approach (implementation feasibility and user acceptance). Thus, we implemented a subset of the design principles, leaving full implementation to future iterations.

Triggers can assume many forms: a gentle vibration on your wrist, an announcement over the radio, a text message on your phone. From the analysis of the available technologies and the viability of the different options, we concluded that text messages are most appropriate in our context because they are amenable to personalization and push communications through the use of smartphones. In the context of a large classroom, where we need to send a significant number of personalized messages, triggering automation is necessary. A messaging bot (i.e., a chatbot) is a software program that can automatically interact with other programs, or with users, via short text - or voice-based messages. Chatbots are popular with college-age individuals [63].

The chatbot we developed leverages the open source Botkit Software Developer Kit (SDK) based on Node.js. After an analysis of the available messaging platforms, we selected Slack. Slack is a communication platform that provides APIs to simplify the development of messaging bots, such as notifications, and interactive/rich messaging (e.g., 
buttons, pictures or files). It is superior to competing platforms (e.g., FB Messenger or Kik) because it does not require personal information (e.g., phone number) upon sign up. To improve the interactivity of the bot we implemented Natural Language Processing (NLP) capabilities through the Wit.ai service. Natural language features, leveraging artificial intelligence techniques, allow users to message the chatbot as they would with human beings. The bot is hosted in an AWS instance running Linux OS and the code on GitHub. Hosting the application on a cloud service frees us from maintaining the server and managing security while enabling computing scalability.

In the first iteration, we implemented a limited number of functionalities with the primary goal of demonstrating the feasibility of the bot messaging system and acceptance by the intended audience of users. During this phase we implemented natural language conversational capabilities to enable students to 1) inquire about approaching deadlines and commitments; 2) engage with the course content.

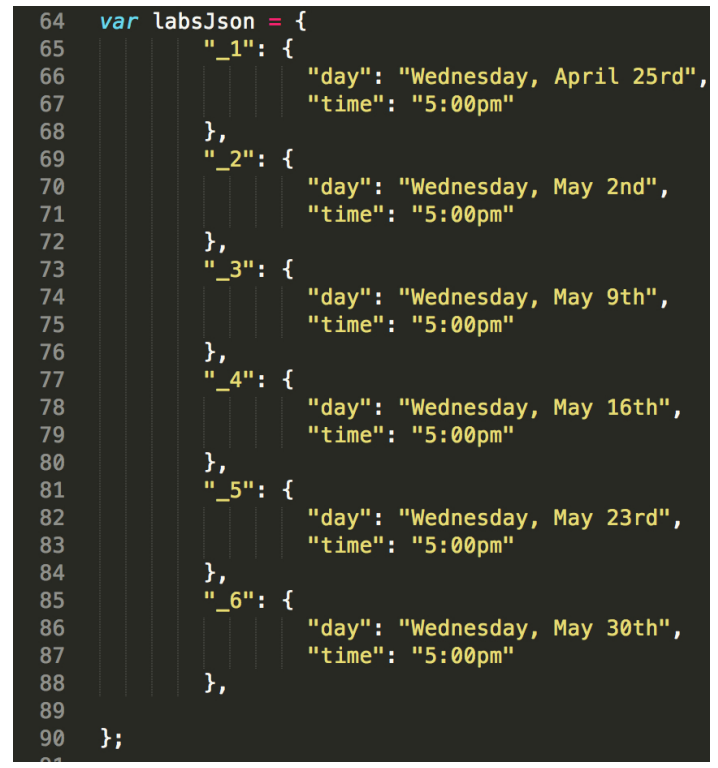

Figure 1. Json containing the checkups' schedule

Students can inquire about schedules regarding class and labs. Furthermore, the chatbot informs students about the day and time of their next practice or exam. For instance, upon messaging the chatbot with a phrase such as "When is the next practice exam?", it will respond "The Excel exam is scheduled for Thursday, May 3". All deadlines and commitments are stored in the JSON format for compatibility with the development platform (Figure 1).

Students can engage with the material by requesting the chatbot to provide a test question. Questions have either a multiple choice or true-false form, and are also stored as JSON files (Figure 1). Questions are randomly selected each time from a large test bank containing of the topics discussed in class (e.g., hardware, software). Answers are also randomized at each request. Random selection at each iteration discourages students from gaming the bot by guessing repeatedly the same question until they get the correct answer (MR1). This is a behavior we had observed with a commercial off-the-shelf system that points students to the specific sentence in the course material that contains the answer to the question when they answer incorrectly. During preliminary research (observations and interviews with students), we noted that students used the testing system to study - rather than as the evaluation and feedback system it was intended to be.

Our engagement bot uses a facilitator trigger to enable students to test their knowledge of the material (MR2). Importantly, however, we designed the bot to focus only on evaluation, not to attempt to overtake the role of the professor in explaining the course concepts. Thus, the chatbot is programmed to provide immediate feedback about the students answer [64]. But feedback is narrowly conceptualized as "information provided by an agent regarding aspects of one's performance or understanding." [64, p.81] Therefore, to maximize the outcomes of feedback, such as increased intrinsic motivation and self-efficacy $[65,20,19]$, when students provide the wrong answer we only inform them that they have not mastered the concept, without disclosing which is the correct answer. We theorize that this approach encourages students to engage more fully with the material and reduces the chances of strategic learning behaviors [18].

Slack, the application managing the users' interaction with the bot, enables users to customize the notification window and the type of messages for which they want to receive a notification (MR3) (e.g., all messages, only direct messages). For instance, users can set custom "do not disturb" windows such that Slack will not send any notifications during the specified time periods.

\section{Evaluation}

The ST artifact focuses on "developing new solutions to know problems" [9, p.345]. Therefore, the evaluation of the ST artifact should be based on its ability to outperform current solutions that addressed our class of problems. As a first step in the evaluation process, we focus on demonstrating the feasibility of our approach [30].

Following the Framework for Evaluation in Design 


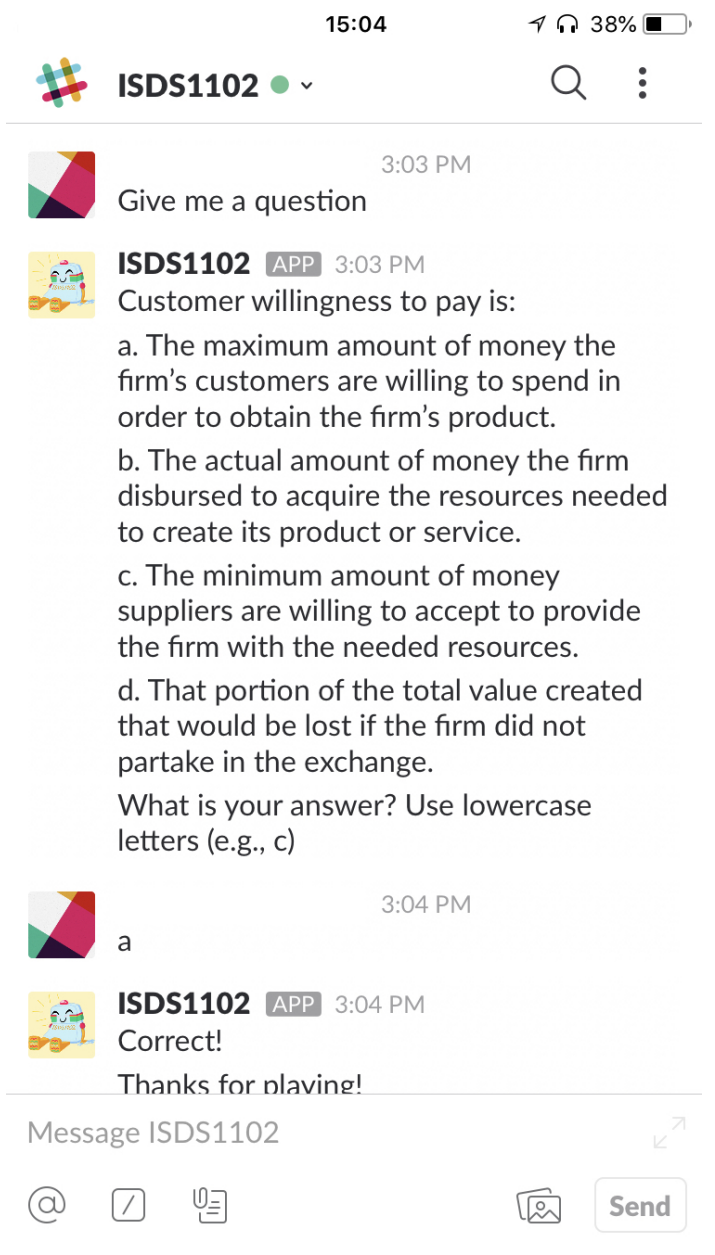

Figure 2. Interaction with the bot

Science (FEDS), we adopt a human risk and effectiveness strategy [33]. This strategy prioritizes multiple formative evaluations early in the design process, to progress rapidly to naturalistic formative evaluations. It is best suited to situations where the ST artifact presents major social risks to its effective implementation, such as the many individual level confounding factors for an initiative seeking to reduce procrastination (e.g., different motivation and prior knowledge levels). It is also optimal when the goal of the research is to establish the utility of the ST artifact in real situations.

A first step in our analysis is to establish the technical and organizational feasibility of the artifact to provide "proof-by-demonstration" [66, p.98] by showing that the ST artifact can be built, implemented, and used by the intended audience.

After an initial formative artificial evaluation of the technical components (e.g., the chatbot), we introduced the ST artifact in a classroom of 34 students. From the beginning of the semester it was evident that students naturally tended to procrastinate. The completion rate for homework was on average $26.23 \%$ during the semester. In keeping with DP1.1 and DP1.2 homework was offered as a service to students, it was not a requirement. However, an analysis of the accesses logs to the online course materials showed evidence of "cramming." Cramming is "a period of neglect of study followed by a concentrated burst of studying immediately before an exam." [67, p.105] In figure 3 , the vertical axis represent the number of page-clicks for the material subject to evaluation. The horizontal axis is a daily time scale spanning the whole semester. The color of the bars represents different topics (i.e., separate book chapters). The black horizontal lines represent the days in which students could schedule examinations at the testing center. The patters show that, on average, students accessed the material during a two-day window prior to the exam. Although worrying, these results were expected based on the literature on college procrastination [27].

Two weeks before the last exam we released the messaging bot. The bot was introduced to students during class via a demo where the instructor explained the available functionalities. Our goal with the pilot study is to assess students' propensity to interact with the bot and to evaluate early consequences of the implementation. Students' propensity to engage with the bot is indicative of how practical it is to implement an additional communication channel (i.e., Slack) in addition to existing ones (e.g., email).

Out of 34 students, 27 completed the course. All of them registered into Slack (100\%). Of the 22 students that took the last test, the one where the bot could be used, 15 interacted at least one time with it. The chatbot was overall well received by those students who used it. From preliminary interviews performed after the final exam students stated "I wish you would have implemented it sooner," while another "I used it over and over to practice for the exam, and when I didn't get the answer right I went back to study the material." However, motivation seemed to play a crucial role in the propensity to use the chatbot as a considerable number of students (33\%) did not interact with it.

A preliminary analysis of performance suggests that the chatbot was instrumental in helping those students who used it perform better in the exam. Although performance in the exam was not a focus of our study, procrastination research indicates that procrastination has moderate, but significant, relationship with classroom learning and grades $(r$ -0.17) [8]. Our results are consistent with these findings. The performance results of the exam show that students 


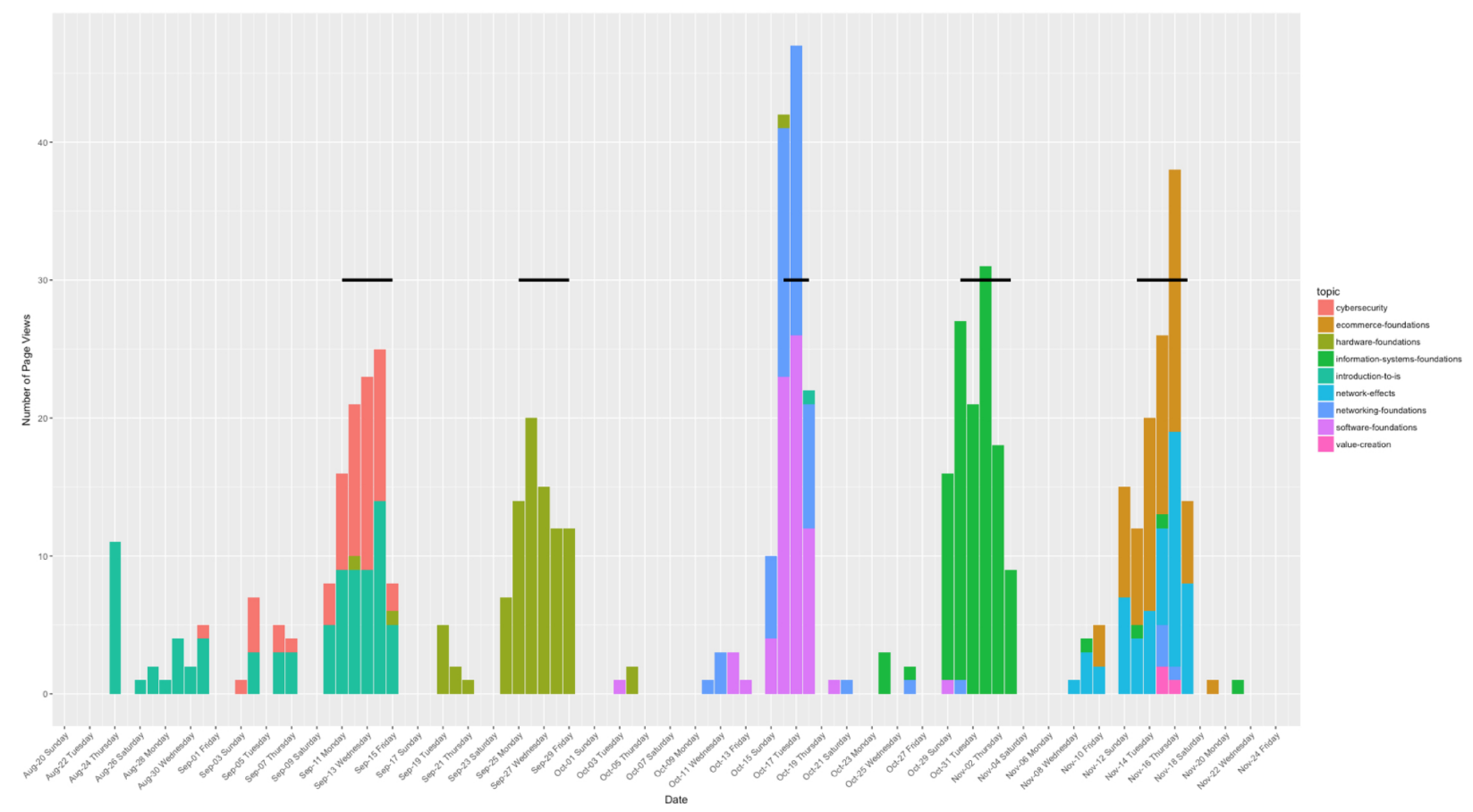

Figure 3. Students' page views in the online application

who interacted at least once with the chatbot outscored those who did not use it by $13.30 \%$, on average. Motivation and conscientiousness likely account for a large portion of the variance in these results. But results are also consistent with the hypothesis of positive chatbot impact. Moreover, in the last exam, students who used the bot performed, on average, $23.38 \%$ better than their own previous average score over the five earlier exams. While the improvement may be due to increased effort during the latter part of the semester, students who did not interact with the bot recorded a lower change (15.33\%). Finally, in the last exam, seven students obtained a perfect score. In the previous five exams, we recorded only six total instances of perfect score across all students and exams.

We do not claim any rigorous conclusions from the pilot results. However, these findings are encouraging. We do demonstrate that the artifact can be built, implemented and, based on the first formative naturalistic evaluation, that the chatbot successfully engages some students with the course material.

\section{Conclusion}

In this paper, we focus our attention on the wicked design problem of reducing academic procrastination. Grounded in the principles of digital nudging, and following the design science research approach, we advance three meta-requirements and nine design principles for an ST artifact that can address this class of problems in the context of large college courses. At this stage, we show "proof-by-demonstration" of the ST artifact by showing that it can be built, implemented and that it is adopted by the intended audience, albeit with varying degrees of interest. Preliminary results of the implementation suggest that the chatbot is effective in engaging some students with the course material and may contribute to students learning and performance.

In keeping with the iterative nature of design science research these preliminary results should be used to refine the design principles and to inform future implementations of the artifact. The next phase in our work is a summative natural evaluation event during the fall semester. The objective is to rigorously corroborate preliminary results using formal interviews and procrastination proxies (e.g., homework completion, spaced access to the materials, performance). We design the next evaluation as a three week long longitudinal field experiment involving 920 students and hope that our early work can stimulate future work in this important area of research and related domains.

\section{References}

[1] D. Kahneman, Attention and effort, vol. 1063. Prentice-Hall Englewood Cliffs, NJ, 1973.

[2] G. Loewenstein and J. Elster, Choice over time. Russell 
Sage Foundation, 1992.

[3] L. Solomon and E. D. Rothblum, "Academic procrastination: Frequency and cognitive-behavioral correlates," vol. 31, pp. 503-509, 101984.

[4] N. A. Milgram, B. Sroloff, and M. Rosenbaum, "The procrastination of everyday life," Journal of Research in Personality, vol. 22, no. 2, pp. 197-212, 1988.

[5] World Health Organization, "Overweight and obesity." http://www. who.int/gho/ncd/risk factors/overweight/en/, 2017. [Last visited on 05/21/2018].

[6] P. Steel, "The nature of procrastination: A meta-analytic and theoretical review of quintessential self-regulatory failure.," Psychological bulletin, vol. 133, no. 1, p. 65, 2007.

[7] A. Ellis and W. Knaus, "Overcoming procrastination: How to think and act rationally in spite of life's inevitable hassles," New York: Institute for Rational Living, 1977.

[8] W. Van Eerde, "A meta-analytically derived nomological network of procrastination," Personality and individual differences, vol. 35, no. 6, pp. 1401-1418, 2003.

[9] S. Gregor and A. R. Hevner, "Positioning and presenting design science research for maximum impact.," MIS quarterly, vol. 37, no. 2, 2013.

[10] S. K. Carpenter, N. J. Cepeda, D. Rohrer, S. H. Kang, and $\mathrm{H}$. Pashler, "Using spacing to enhance diverse forms of learning: Review of recent research and implications for instruction," Educational Psychology Review, vol. 24, no. 3, pp. 369-378, 2012.

[11] D. M. Tice and R. F. Baumeister, "Longitudinal study of procrastination, performance, stress, and health: The costs and benefits of dawdling," Psychological science, vol. 8, no. 6, pp. 454-458, 1997.

[12] B. W. Tuckman, "The development and concurrent validity of the procrastination scale," Educational and psychological measurement, vol. 51, no. 2, pp. 473-480, 1991.

[13] L. J. Solomon and E. D. Rothblum, "Academic procrastination: Frequency and cognitive-behavioral correlates.," Journal of counseling psychology, vol. 31, no. 4, p. 503, 1984.

[14] A. H. Chun Chu and J. N. Choi, "Rethinking procrastination: Positive effects of" active" procrastination behavior on attitudes and performance,' The Journal of social psychology, vol. 145, no. 3, pp. 245-264, 2005.

[15] Y. E. Kalay, "Virtual learning environments," Journal of Information Technology in Construction (ITcon), vol. 9, no. 13, pp. 195-207, 2004.

[16] R. M. Bernard, P. C. Abrami, E. Borokhovski, C. A. Wade, R. M. Tamim, M. A. Surkes, and E. C. Bethel, "A meta-analysis of three types of interaction treatments in distance education," Review of Educational research, vol. 79, no. 3, pp. 1243-1289, 2009.

[17] E. Gebre, A. Saroyan, and R. Bracewell, "Students' engagement in technology rich classrooms and its relationship to professors' conceptions of effective teaching," British Journal of Educational Technology, vol. 45, no. 1, pp. 83-96, 2014.

[18] K. Bain, What the best college teachers do. Harvard University Press, 2011.
[19] E. Deci and R. M. Ryan, Intrinsic motivation and self-determination in human behavior. Springer Science \& Business Media, 1985.

[20] R. M. Ryan and E. L. Deci, "Self-determination theory and the facilitation of intrinsic motivation, social development, and well-being.," American psychologist, vol. 55, no. 1, p. 68, 2000.

[21] G. Piccoli, J. Rodriguez, B. Palese, and M. Bartosiak, "The dark side of digital transformation: The case of information systems education," in International Conference of Information Systems, 2017.

[22] M. Y. Vardi, "Will moocs destroy academia?," Communications of the ACM, vol. 55, no. 11, pp. 5-5, 2012.

[23] G. Piccoli, R. Ahmad, and B. Ives, "Web-based virtual learning environments: A research framework and a preliminary assessment of effectiveness in basic it skills training," MIS quarterly, pp. 401-426, 2001.

[24] E. C. Kokkelenberg, M. Dillon, and S. M. Christy, "The effects of class size on student grades at a public university," Economics of Education Review, vol. 27, no. 2, pp. 221-233, 2008.

[25] O. Bandiera, V. Larcinese, and I. Rasul, "Heterogeneous class size effects: New evidence from a panel of university students," The Economic Journal, vol. 120, no. 549, pp. 1365-1398, 2010.

[26] D. F. Chambliss, How college works. Harvard University Press, 2014.

[27] G. Schraw, T. Wadkins, and L. Olafson, "Doing the things we do: A grounded theory of academic procrastination.," Journal of Educational psychology, vol. 99, no. 1, p. 12, 2007.

[28] M. K. Sein, O. Henfridsson, S. Purao, M. Rossi, and R. Lindgren, "Action design research," MIS quarterly, pp. 37-56, 2011.

[29] M. S. Silver and M. L. Markus, "Conceptualizing the sociotechnical (st) artifact," Systems, Signs \& Actions, vol. 7, no. 1, pp. 82-89, 2013.

[30] A. R. Hevner, S. T. March, J. Park, and S. Ram, "Design science in information systems research," MIS Quarterly, vol. 28, no. 1, 2004.

[31] K. Peffers, T. Tuunanen, M. A. Rothenberger, and S. Chatterjee, "A design science research methodology for information systems research," Journal of management information systems, vol. 24, no. 3, pp. 45-77, 2007.

[32] J. G. Walls, G. R. Widmeyer, and O. A. El Sawy, "Building an information system design theory for vigilant eis," Information systems research, vol. 3 , no. 1, pp. 36-59, 1992.

[33] J. Venable, J. Pries-Heje, and R. Baskerville, "Feds: a framework for evaluation in design science research," European Journal of Information Systems, vol. 25, no. 1, pp. 77-89, 2016.

[34] P. J. Ågerfalk, "Insufficient theoretical contribution: a conclusive rationale for rejection?," 2014.

[35] J. L. Johnson and A. M. Bloom, "An analysis of the contribution of the five factors of personality to variance in academic procrastination," Personality and Individual Differences, vol. 18, no. 1, pp. 127-133, 1995. 
[36] C. Lay, A. Kovacs, and D. Danto, "The relation of trait procrastination to the big-five factor conscientiousness: an assessment with primary-junior school children based on self-report scales," Personality and Individual Differences, vol. 25, no. 2, pp. 187-193, 1998.

[37] A. Bandura, "Self-efficacy: toward a unifying theory of behavioral change.," Psychological review, vol. 84, no. 2, p. $191,1977$.

[38] R. M. Klassen, L. L. Krawchuk, and S. Rajani, "Academic procrastination of undergraduates: Low self-efficacy to self-regulate predicts higher levels of procrastination," Contemporary Educational Psychology, vol. 33, no. 4, pp. 915-931, 2008.

[39] R. M. Klassen, R. P. Ang, W. H. Chong, L. L. Krawchuk, V. S. Huan, I. Y. Wong, and L. S. Yeo, "A cross-cultural study of adolescent procrastination," Journal of research on Adolescence, vol. 19, no. 4, pp. 799-811, 2009.

[40] L. A. Haycock, P. McCarthy, and C. L. Skay, "Procrastination in college students: The role of self-efficacy and anxiety," Journal of counseling \& development, vol. 76, no. 3, pp. 317-324, 1998.

[41] D. H. Schunk, "Self-efficacy and academic motivation," Educational psychologist, vol. 26, no. 3-4, pp. 207-231, 1991.

[42] I. Katz, K. Eilot, and N. Nevo, "I'll do it later: Type of motivation, self-efficacy and homework procrastination," Motivation and Emotion, vol. 38, no. 1, pp. 111-119, 2014.

[43] P. Steel, The procrastination equation: How to stop putting things off and start getting stuff done. Random House Canada, 2010.

[44] Y.-G. Lin, W. J. McKeachie, and Y. C. Kim, "College student intrinsic and/or extrinsic motivation and learning," Learning and individual differences, vol. 13, no. 3, pp. 251-258, 2003.

[45] E. L. Deci, R. Koestner, and R. M. Ryan, "A meta-analytic review of experiments examining the effects of extrinsic rewards on intrinsic motivation.", Psychological bulletin, vol. 125, no. 6, p. 627, 1999.

[46] M. Magni, M. S. Taylor, and V. Venkatesh, "to play or not to play: A cross-temporal investigation using hedonic and instrumental perspectives to explain user intentions to explore a technology," International journal of human-computer studies, vol. 68, no. 9, pp. 572-588, 2010.

[47] R. Santhanam, D. Liu, and W.-C. M. Shen, "Research notegamification of technology-mediated training: Not all competitions are the same," Information Systems Research, vol. 27, no. 2, pp. 453-465, 2016.

[48] S. Thiebes, S. Lins, and D. Basten, "Gamifying information systems-a synthesis of gamification mechanics and dynamics," in European Conference of Information Systems, 2014.

[49] E. L. Deci and R. M. Ryan, "The" what" and" why" of goal pursuits: Human needs and the self-determination of behavior," Psychological inquiry, vol. 11, no. 4, pp. 227-268, 2000.

[50] J. E. Kemp, G. R. Morrison, and S. M. Ross, "Designing effective instruction," New York: Merrill, 1994.

[51] M. F. Young, S. Slota, A. B. Cutter, G. Jalette, G. Mullin, B. Lai, Z. Simeoni, M. Tran, and M. Yukhymenko, "Our princess is in another castle: A review of trends in serious gaming for education," Review of educational research, vol. 82, no. 1, pp. 61-89, 2012.
[52] R. Thaler and C. Sunstein, Nudge: Improving Decisions about Health, Wealth, and Happiness. Yale University Press, 2008.

[53] R. Thaler and C. Sunstein, "Libertarina paternalism is not an oxymoron," University of Chicago Public Law and Legal Theory Working Paper, no. 43, 2003.

[54] M. Weinmann, C. Schneider, and J. vom Brocke, "Digital nudging," Business \& Information Systems Engineering, vol. 58, no. 6, pp. 433-436, 2016.

[55] R. Thaler, C. Sunstein, and J. Balz, Choice Architecture, pp. 428-439. Princeton University Press, 2012.

[56] Y. Yoo, "Computing in everyday life: A call for research on experiential computing," MIS quarterly, pp. 213-231, 2010.

[57] B. J. Fogg, "A behavior model for persuasive design," in Proceedings of the 4th international Conference on Persuasive Technology, p. 40, ACM, 2009.

[58] J. Martin, S. Edwards, and C. Shaffer, "The effects of procrastination interventions on programming project success," in Proceedings of the eleventh annual International Computing Education Research (ICER) Conference, pp. 3-11, ACM, 2015.

[59] S. S. Intille et al., "Ubiquitous computing technology for just-in-time motivation of behavior change.," Medinfo, vol. 107, pp. 1434-1437, 2004.

[60] D. I. Cordova and M. R. Lepper, "Intrinsic motivation and the process of learning: Beneficial effects of contextualization, personalization, and choice.,"Journal of educational psychology, vol. 88, no. 4, p. 715, 1996.

[61] H. Fan and M. S. Poole, "What is personalization? perspectives on the design and implementation of personalization in information systems," Journal of Organizational Computing and Electronic Commerce, vol. 16, no. 3-4, pp. 179-202, 2006.

[62] P. Brusilovski, A. Kobsa, and W. Nejdl, The adaptive web: methods and strategies of web personalization, vol. 4321. Springer Science \& Business Media, 2007.

[63] Forbes, "Chatbots And Millennials: How Smart Brands Should Be Using Artificial Intelligence." https:// go०.gl/3yb2dS, 2016. [Last visited on 06/07/2018].

[64] J. Hattie and H. Timperley, "The power of feedback," Review of educational research, vol. 77 , no. 1 , pp. 81-112, 2007.

[65] D. H. Schunk, "Effects of effort attributional feedback on children's perceived self-efficacy and achievement.," Journal of educational psychology, vol. 74 , no. 4, p. 548 , 1982.

[66] J. F. Nunamaker Jr, M. Chen, and T. D. Purdin, "Systems development in information systems research," Journal of management information systems, vol. 7, no. 3, pp. 89-106, 1990.

[67] R. Sommer, "The social psychology of cramming," Journal of Counseling \& Development, vol. 47, no. 2, pp. 104-109, 1968. 